\title{
microRNA-150-5p: A Novel Blood-Based Biomarker for Alzheimer's Dementia with Good Correlation to Cognition, Cerebrospinal Fluid Amyloid- $\beta$, and Cerebral Atrophy
}

\section{Sook-Yoong Chia}

National Neuroscience Institute

\section{Ashwati Vipin}

National Neuroscience Institute

Kok-Pin Ng

National Neuroscience Institute

Haitao Tu

National Neuroscience Institute

Ananth Bommakanti

Temasek Life Sciences Laboratory Ltd

\section{Brian Zhiyang Wang}

National Neuroscience Institute

Yi Jayne Tan

National Neuroscience Institute

\section{Fatin Zahra Zailan}

National Neuroscience Institute

\section{Adeline Su-Lyn Ng}

National Neuroscience Institute

\section{Shuo-Chian Ling}

National University Singapore Yong Loo Lin School of Medicine

Katsutomo Okamura

Nara Institute of Science and Technology: Nara Sentan Kagaku Gijutsu Daigakuin Daigaku

Eng-King Tan

National Neuroscience Institute

Nagaendran Kandiah

National Neuroscience Institute

\section{Li Zeng ( $\nabla$ li_zeng@nni.com.sg)}

National Neuroscience Institute https://orcid.org/0000-0003-3242-2286 
Research

Keywords: Alzheimer's disease, Biomarker, CSF, MRI, MicroRNA.

Posted Date: October 11th, 2021

DOl: https://doi.org/10.21203/rs.3.rs-942370/v1

License: (c) (i) This work is licensed under a Creative Commons Attribution 4.0 International License. Read Full License 


\section{Abstract}

Background: There is an urgent need for non-invasive, cost-effective biomarkers for Alzheimer's disease (AD), such as blood-based biomarkers. It is not only to support clinical diagnosis of dementia, but also to allow for timely pharmacological and non-pharmacological interventions evaluation. The aim of this study is to identify and validate a novel blood-based microRNA (miRNA) biomarker for dementia of Alzheimer's disease type (DAT). The miRNA correlations with AD pathology and AD clinical-radiological imaging were conducted.

Methods: We conducted miRNA-sequencing (miRNA-Seq) using peripheral blood mononuclear cells (PBMCs) isolated from a discovery cohort comprising DAT, mild cognitive impairment (MCl), and healthy subject (HS). Identified miRNA was validated in an independent cohort. Correlation analysis evaluated the relationships between miRNA expression and DAT clinical measures, including Mini-Mental State Examination (MMSE) and Montreal Cognitive Assessment (MoCA) scores, CSF A $\beta_{1-42}$ and tau levels, and AD pattern cerebral atrophy. Furthermore, we conducted bioinformatics analysis and cell-based assay to identify miRNA target genes.

Results: MiRNA-seq identified a distinct miRNA (miR-328-3p, miR-7706, and miR-150-5p) expression signature differentiating DAT from $\mathrm{MCl}$ and HS. qPCR analysis reveals that miR-150-5p was consistent with the miRNA-seq data and was further validated. Specifically, we found that miR-150-5p expression was significantly upregulated in DAT compared to $\mathrm{MCl}$ and $\mathrm{HS}$, and discriminated DAT from $\mathrm{MCl}$ and $\mathrm{HS}$ with a high accuracy with AUC of 0.86 and 0.86 , respectively. We further found that higher miR-150-5p levels correlated with clinical measures of DAT, including lower global cognitive scores of MMSE and MoCA, lower CSF $A \beta_{1-42}$, and higher CSF tau. Interestingly, we observed that higher miR-150-5p levels is associated with the lower grey matter volumes in the medial temporal lobe, posterior cingulate cortex and precuneus. These regions implicated default mood network and executive control network regions that are important for $A D$ brain atrophy. Furthermore, pathway analysis identified the targets of miR-150-5p to be enriched in the Wnt signalling pathway, including programmed cell death 4 (PDCD4). We further found that PDCD4 was downregulated in DAT blood and was downregulated by miR-150-5p at both transcriptional and protein levels.

Conclusions: Our findings demonstrated that miR-150-5p is a reliable clinical blood-based biomarker for DAT.

\section{Introduction}

The advancement of biomarker research in Alzheimer's disease (AD) has shifted the diagnosis of AD from a clinical syndrome to a biological construct [1]. However, the current AD biomarkers such as CSF amyloid- $\beta$ 1-42 (A $\left.\beta_{1-42}\right)$ and phosphorylated tau, as well as amyloid and tau PET are limited by either invasiveness or cost and hence are available only in very few academic centres [2-4]. As such, it is 
imperative to develop a non-invasive and inexpensive blood-based biomarker that is specific for $A D$ pathophysiology for clinical application in detecting $A D$ in wider populations.

MicroRNAs (miRNAs) are small, conserved non-coding RNAs that modulate post-transcription gene expression by suppressing specific target miRNAs $[5,6]$. miRNAs play vital roles in the body's essential processes including cell proliferation, cell development and stress responses amongst others. In the nervous system, miRNAs are also widespread and regulate key functions such as neurite outgrowth, dendritic spine morphology, neuronal differentiation, and synaptic plasticity $[7,8]$ There has been increasing research interest in the relationship between miRNAs and neurocognitive disorders following the discovery of miRNAs dysregulation in neurocognitive disorders. Specifically, miRNAs dysfunction has been shown in mild cognitive impairment $(\mathrm{MCl})$ and Alzheimer's disease dementia although they lack correlation with clinical phenotypes [9-13]. It has been shown that dysregulation of miRNAs in AD affects $A \beta_{1-42}$ and phosphorylated tau production, increases oxidative stress and neuroinflammation [14, 15].

Circulating miRNAs in whole blood, plasma, serum, CSF, or peripheral blood mononuclear cells (PBMC) are increasingly being examined as candidates of blood-based AD biomarkers [16-18]. A recent systematic review included 20 studies reported the potential for 102 dysregulated miRNAs in peripheral blood of $A D$ patients as potential biomarker for $A D$ [19]. With respect to miRNA dysregulation in the human brain, the same review reported the identification of 250 miRNAs from 27 studies. When crossreferencing the miRNAs from peripheral blood and the brain, 47 common miRNAs were found, of which 30 miRNAs were dysregulated in the brain tissue with known Braak Stages. Among these 30 miRNAs, 10 of them were observed to be dysregulated at Braak Stage III, and their expression levels in peripheral blood and the brain correlated with each other (miR-30e, miR-34c, miR-107, miR-200c, miR-210, miR-485) while others had opposing levels (miR-26b, miR-34a, miR-125b, miR-146a). Taken together, miRNAs do have the potential and show promise to be incorporated in clinical practice for diagnosis of $A D$, and for monitoring patients receiving preventive and therapeutic interventions. However, more studies need to be devoted to identifying correlations with current validated $A D$ biomarkers to establish whether or not a particular miRNA can be considered a specific biomarker of AD. However, reliable miRNA-based tests to complement and support the clinical diagnosis of Alzheimer's disease is lacking. Therefore, studies focusing on the correlations of miRNAs with current Alzheimer's disease biomarkers are paramount.

In this study, we evaluated PBMC as a source of miRNA as 1) it's a major cellular component of the human immunity; 2) stable levels in bloodstream; 3 ) can be obtained using minimally invasive procedure; 4) low cost and 5) low risk of being contaminated when compared to miRNAs extracted from other body fluids [20]. Here, in a discovery and an independent cohort of individuals with dementia of Alzheimer's disease type (DAT), mild cognitive impairment ( $\mathrm{MCl}$ ) and healthy individuals (HS), we profiled the expression of PBMC-derived miRNAs using high throughput miRNA-seq. We aimed to identify and validate miRNA that is specific for DAT. We further correlated the miRNA with AD clinical biomarkers and studied its target gene in biological pathways. 


\section{Methods}

\section{Study participants}

Participants with mild DAT and $\mathrm{MCl}$ were recruited from a tertiary neurology center (National

Neuroscience Institute, Singapore) while HS were recruited from the community between 2015-2018. The diagnoses of DAT and $\mathrm{MCl}$ were made using the NIA-AA criteria $[2,16]$ and supported by a Clinical Dementia Rating [21] (CDR) score of 1 and 0.5 respectively. DAT participants were in the mild stage of dementia as reflected by the CDR score of 1 and Mini-Mental State Examination (MMSE) [22], as well as Montreal Cognitive Assessment (MoCA) [23] scores being in the mild impairment range. All individuals received a consensus diagnosis from a panel comprising cognitive neurologists, psychologists, and specialist nurses. Key exclusion criteria included serious neurological or psychiatric comorbidities, diagnosis of dementia with Lewy bodies (DLB), vascular dementia (VAD) and frontotemporal dementia (FTD) as well as a history of alcohol or drug abuse.

The medical history and demographic characteristics of the study participants were collected via a standardized interview. Study participants underwent a standardized assessment of their global cognition using the MMSE [22] and MoCA [23], blood test, lumbar puncture for CSF analysis [24] and a 3T-MRI scan within six months of clinical and neuropsychological evaluations.

\section{MiRNA extraction and sequencing}

Extraction of PBMC from whole blood was performed as described by Maes OC et al., [25] with slight modification. Following extraction, PBMC were resuspended in QIAzol Lysis Reagent (Qiagen) and stored at $-80^{\circ} \mathrm{C}$ until further use. Total RNAs including miRNA was extracted from PBMC using miRNeasy Mini Kit (Qiagen) following manufacturer's instructions. Concentration and Quality of extracted RNAs were measured using NanoDrop1500 (Thermo Fisher Scientific). RNA integrity number (RIN) of RNAs was assessed by Bioanalyser, where RNA samples with RIN $\geq 8.0$ and RNA ration [28s/18s]: $\geq 1.5$ were used for library preparation and miRNA-seq.

The small RNA libraries were prepared by Beijing Genomics Institute (BGI) using the TruSeq small RNA kit (Illumina). During this analysis, all the runs were considered as independent. The sequences were assessed for quality using FASTQC. The Primer, adapter and contaminant sequences were removed using Cutadapt/TRIMMOMATIC. Read lengths shorter than 15 nucleotides were removed. The cleaned sequences were collapsed using the FastX-toolKit (fastX collapser) and reduced to unique reads. The subsequent file was processed using miRDEEP2 pipeline. The reads were mapped to the human genome (hg19) and precursor miRNA sequences for miRbase version 21. The final output of the count file summarizing the mapped miRNAs for each sample was used for further analysis.

\section{Validation of microRNA by qPCR}

Differentially expressed miRNAs were validated using Taqman MicroRNA Assays (Applied Biosystem). Each Assay contains miRNA-specific Taqman minor groove binder (MGB) probe and PCR primers for hsa- 
miR-150-5p, hsa-miR-150-3p, hsa-miR-328-3p, hsa-miR-7706 and along with U6 small nuclear RNA (U6) which served as a reference control for miRNA expression normalization. MiRNA expression changes were calculated relative to U6 using the $2^{-\Delta \Delta \mathrm{Ct}}$ method.

\section{MRI image acquisition and pre-processing}

High resolution T1-weighted MPRAGE (Magnetization Prepared Rapid Gradient Echo) sequences performed on a 3T Prisma Fit System (Siemens, Erlangen, Germany) were obtained as follow: 192 continuous sagittal slices, TR/TE $/ \mathrm{TI}=2300 / 2.28 / 900 \mathrm{~ms}$, flip angle $=8 \circ$, FOV $=256 \times 240 \mathrm{~mm} 2$, matrix $=$ $256 \times 240$, isotropic voxel size $=1.0 \times 1.0 \times 1.0 \mathrm{~mm} 3$, bandwidth $=200 \mathrm{~Hz} /$ pixel. We used the Computational Anatomy Toolbox (http://dbm.neuro.uni-jena.de/cat12/) protocol in Statistical Parametric Mapping (SPM12) (http://Page | 6www.fil.ion.ucl.ac.uk/spm/), to process the T1 images for voxel-based morphometry (VBM) analysis. Specifically, all 3D T1-weighted MRI scans were normalized using an affine transformation followed by non-linear registration, corrected for bias field in homogeneities. Images were then segmented to derive subject-level GM, WM, and CSF components [26]. The Diffeomorphic Anatomic Registration Through Exponentiated Lie algebra algorithm (DARTEL) was used to normalize the segmented scans into the standard MNI space which provides better precision in spatial normalization to the template [27]. All obtained segmented, modulated, and normalized GM and WM images were then smoothed using an 8-mm full-width-half-maximum isotropic Gaussian smoothing kernel.

\section{MRI regions of interest derivation}

We also applied a multiple seed-based approaches to test the association between GMV and miRNA levels specifically in regions of interest (ROIs) belonging to the Default mode network (DMN) and executive control network (ECN). We selected nine ROIs covering the DMN and ECN based on a prior study [28]. The DMN ROIs included the left and right parahippocampal cortex (L and R HIP), medial prefrontal cortex (mPFC), posterior cingulate cortex (PCC) and precuneus (PCN) and the ECN ROIs included the left and right dorsolateral prefrontal cortex ( $L$ and $R$ DLPFC) and the left and right posterior parietal cortex ( $L$ and R PPC) in standard space. Average GMV from these network ROls were derived using the MarsBar toolbox in SPM12. Following derivation of miRNA levels, a median split analysis was carried out for each of the miRNAs. A binary assignment was formulated with participants less than the median level of miRNA levels were assigned as " 0 " and those with greater than median levels of miRNAs were assigned " 1 ".

\section{CSF processing for $A \beta_{1-42}$ and tau measurement}

CSF was collected via lumbar puncture then centrifuged at $2000 \mathrm{~g}$ for $10 \mathrm{~min}$ at $4^{\circ} \mathrm{C}$. The resulting supernatant was aliquoted and stored at $-80^{\circ} \mathrm{C}$ until further analysis. CSF levels of $A \beta_{1-42}$ and tau were measured using the INNOTEST ® (Fujirebio Europe NV) according to the manufacturer's instructions [29]. A standard protocol for collection of CSF and measurement of $A \beta_{1-42}$ and tau was used for all participants.

\section{Plasmid Constructs}


pmirGLO- PDCD4 -3'UTR: A 1,236 bp DNA fragment was amplified from human PDCD4 cDNA (NCBI reference number: NM_014456.5) 3'UTR region containing four miR-150-5p predictive binding sites using primer set: 5'-CTAGCTAGCATGTTGGCTGCTGCTGTTGAGATAC-3' and 5'-

ACGGTCGACTGCCTCCCAGGTTCAAGCAATTCT-3'. The purified DNA fragment was then digested with Nhel and Sall restriction enzymes and inserted into the pmirGLO vector (Promega) [12,13]. The sequence of formed plasmid was confirmed by Sanger sequencing.

\section{Luciferase assay}

Human embryonic kidney (HEK)293T cells were cultured in 6-well plates prior transfection. Cells were cotransfected with miR-150-5p mimic and empty vector or PDCD4 3'UTR plasmid using lipofectamine 2000 (Invitrogen) according to manufacturer's protocol. Cells were harvested 48 hours post transfection and subjected to measure respective luciferase activity with the Dual Luciferase Reporter Assay Kit (Promega) by using a GloMAX 20/20 luminometer (Promega).

\section{mRNA Reverse transcription and qPCR}

Total mRNAs were reverse-transcribed by iScript ${ }^{\mathrm{TM}}$ cDNA Synthesis kit (Bio-rad) and generated cDNA was subjected to qPCR using All in One ${ }^{T M}$ qPCR Mix (Genecopoeia). $\beta$-actin was used as a reference gene for mRNA expression normalization. Relative expression of the genes was calculated using the $2^{-\triangle \Delta C T}$ method relative to values of $\beta$-actin.

\section{Western blot analysis}

Proteins were extracted from cells using RIPA lysis buffer supplemented with protease inhibitor cocktail (MedChemExpress) and phosphatase inhibitor cocktail (MedChemExpress). Protein concentrations were measured using RC-DC protein assay kit II (Bio-Rad). $20 \mu \mathrm{g}$ of protein lysates were separated by SDSPAGE and then transferred to polyvinylidene fluoride membrane (PVDF membrane, Millipore). The membranes were blocked with 5\% milk in tris buffered saline with tween 20 (TBST) for 1 hour at room temperature and incubated with a 1:1000 dilution of primary antibodies (rabbit anti- $P D C D 4$, Merck; mouse anti- $\beta$-actin, Santa Cruz) at $4^{\circ} \mathrm{C}$ overnight. The membranes were then washed with TBST, followed by incubation of horseradish peroxidase (HRP)-conjugated secondary antibody (1:3000, GE Healthcare). Protein bands were visualized by Pierce ECL Western Blotting Substrate (Thermofisher Scientific). Band intensity was quantified using ImageJ software.

\section{Statistical analysis}

\section{Statistical analysis for evaluation and correlation}

Evaluation of miRNA, mRNA and protein expression levels, ROC curves, and Pearson correlation were analysed with GraphPad Prism 6.0. One-way ANOVA with Tukey's multiple comparisons test was used for evaluation of expression levels and values were expressed as mean \pm SEM. Differences between the study participants were considered statistically significant at $p<0.05$.

\section{Voxel-based morphometry and region of interest analyses}


Group differences in grey matter volume (GMV): To examine group differences, we built a voxel-wise Analysis of Covariates (ANCOVA) general linear model with GMV as the dependent variable, binary group membership as either below or above median miRNA levels with age and gender as nuisance covariates. The GM regions which showed significant group differences based on miRNA median split were examined using a threshold of Family-wise error correction of $p<0.05$ or an uncorrected $p<0.001$ and a minimum cluster size of 100 voxels [30,31]. Significant clusters in the GM were anatomically identified using the Automated Anatomical Labelling atlas labels.

Associations between default mode network and executive control network regions of interest GMV and miRNA levels: Pearson's correlation analysis was used to assess the association between ROI GMV and miRNA levels. Partial Pearson's correlation analysis was used to assess the association between ROI GMV and miRNA levels after controlling for age at visit as a covariate. Multiple comparisons correction across the nine ROls was conducted using FWE-correction at $p<0.0055$.

\section{Statistical analysis for bioinformatics}

All the miRNAs with reading counts $<50$ summed up for all samples for each group (HS/MCl/DAT individuals) were removed. The read counts were normalized using Quantile Normalization or the raw file was analyzed for differentially regulated miRNA using DESEQ2 pipeline (TMM Normalization) or using SAMSEQ pipeline (Quantile Normalization) or Quantile normalized data. $P$-values were calculated using Wilcoxon-Mann-Whitney (WMW) test. The adjusted $p$ values (FDR) were calculated using BenjaminiHochberg approach. For hierarchical classification and K means clustering approach e1701 and caret packages were used in $\mathrm{R}$.

\section{Results}

\section{MiRNA-sequencing reveals an expression signature that distinct DAT from $\mathrm{MCl}$ and $\mathrm{HS}$}

We performed Hiseq2000 microRNA-sequencing (miRNA-seq) in the PBMCs of age matched discovery cohort of $19 \mathrm{HS}, 16 \mathrm{MCl}$, and 19 DAT individuals (Table 1). A total of 956 mature miRNAs originating from 739 unique precursors met the expression cut-off (normalized read counts $>50$ ). These samples showed similar read count distributions for individual genes and were virtually identical after Quantile Normalization, confirming successful normalization. To determine distinctive miRNA signatures among $\mathrm{HS}, \mathrm{MCl}$, and DAT subjects, we performed sample clustering analysis using the hierarchical clustering approach. Four distinct clusters of miRNA expressions were observed, with DAT being enriched in one of them but none in $\mathrm{MCl}$ and $\mathrm{HS}$ (Fig. 1a). This indicates that there is a distinct miRNA expression signature that differentiates DAT from $\mathrm{MCl}$ subjects, and DAT from HS subjects. 
Table 1

Characteristics of discovery cohort.

\begin{tabular}{|c|c|c|c|c|}
\hline $\begin{array}{l}\text { Discovery } \\
\text { cohort }\end{array}$ & $\begin{array}{l}\text { Healthy Subjects } \\
\text { (HS) }\end{array}$ & $\begin{array}{l}\text { Mild cognitive } \\
\text { impairment (MCl) }\end{array}$ & $\begin{array}{l}\text { Alzheimer's dementia } \\
\text { (DAT) }\end{array}$ & $P$-value \\
\hline $\begin{array}{l}\text { Sample } \\
\text { numbers }\end{array}$ & 19 & 16 & 19 & \\
\hline Gender & 10 (53\%) & $5(31 \%)$ & $9(47 \%)$ & 0.425 \\
\hline Male & $9(47 \%)$ & 11 (69\%) & 10 (53\%) & \\
\hline \multicolumn{5}{|l|}{ Female } \\
\hline Age, years & $65.6 \pm 6.5$ & $64.4 \pm 7.3$ & $67.7 \pm 6.3$ & 0.226 \\
\hline $\begin{array}{l}\text { Education, } \\
\text { years }\end{array}$ & $12.1 \pm 2.2$ & $11.6 \pm 3.1$ & $8.6 \pm 3.1$ & $<.001^{* * *+}$ \\
\hline MMSE score & $29.1 \pm 1.2$ & $27.4 \pm 1.9$ & $23.1 \pm 4.4$ & $\begin{array}{l}<.001^{\star \star \star}+\# \\
\end{array}$ \\
\hline MoCA score & $27.5 \pm 1.9$ & $25.1 \pm 2$ & $20.5 \pm 5.0$ & $\begin{array}{l}< \\
0.001^{\star \star \star} \dagger\end{array}$ \\
\hline \multicolumn{5}{|c|}{$\begin{array}{l}\text { Values are represented as mean } \pm S D \text { or number (\%). Demographic characteristics were compared } \\
\text { between disease group using the } \chi^{2} \text { test for gender, and Kruskal-Wallis with Dunn's test for numerical } \\
\text { variables. } \star \star \star p<0.001 \text { for DAT vs HS subjects; } \dagger p<0.05 \text { for DAT vs MCl subjects; } \# p<0.05 \text { for MCl } \\
\text { vs HS subjects. }\end{array}$} \\
\hline
\end{tabular}

Volcano plot further revealed that there is a distinct miRNA expression signature between DAT and HS (Fig. 1b), DAT and $\mathrm{MCl}$ (Fig. 1C); while the miRNA expression was indistinguishable between $\mathrm{HS}$ and $\mathrm{MCl}$ (Fig. 1d). Specifically, when comparing DAT with HS, miR-328-3p (Fig. 1e) and miR-7706 (Fig. 1f) were found to be downregulated in DAT, whereas miR-150-5p were found to be highly upregulated in DAT (Fig. 1g). Our findings suggested a distinct miRNA expression signature differentiating DAT from $\mathrm{MCl}$ and HS. miR-150-5p was selected for further evaluation in another independent cohort because miR-150-5p have been found to be upregulated in the postmortem brain tissue of $A D$ patients by next generation sequencing[32]. Further investigation will warrant the role of miR-150-5p on $A D$

\section{MiR-150-5p expression is upregulated in DAT}

We validated the miR-150-5p in an independent cohort comprising $16 \mathrm{HS}, 27 \mathrm{MCl}$, and 21 DAT (Table 2) and found that the expression of miR-150-5p was significantly upregulated by $76.9 \%$ in DAT compared to $\mathrm{HS}$, and by $73.3 \%$ compared to $\mathrm{MCl}$ individuals (Fig. 2a). This finding was consistent with earlier miRNAseq data that miR-150-5p was highly expressed in DAT (Fig. 1g). Moreover, findings from the AUC analysis showed that miR-150-5p levels discriminated DAT from MCl and HS individuals with a good accuracy of AUC 0.86 (95\% Cl 0.76-0.96) and AUC 0.86 (95\% Cl 0.73-0.99) respectively (Fig. 2b). However, miR-150-5p levels did not discriminate $\mathrm{MCl}$ from HS individuals with an AUC of 0.53 (95\% $\mathrm{Cl} 0.33-0.72$ ). 
Table 2

Characteristics of an independent cohort.

\begin{tabular}{|c|c|c|c|c|}
\hline $\begin{array}{l}\text { Independent } \\
\text { Cohort }\end{array}$ & $\begin{array}{l}\text { Healthy } \\
\text { Subjects (HS) }\end{array}$ & $\begin{array}{l}\text { Mild cognitive } \\
\text { impairment (MCl) }\end{array}$ & $\begin{array}{l}\text { Alzheimer's } \\
\text { dementia (DAT) }\end{array}$ & $P$-value \\
\hline $\begin{array}{l}\text { Samples } \\
\text { numbers }\end{array}$ & 16 & 27 & 21 & \\
\hline Gender & $10(62 \%)$ & $19(70 \%)$ & $9(43 \%)$ & 0.15 \\
\hline Male & $6(38 \%)$ & $8(30 \%)$ & 12 (57\%) & \\
\hline \multicolumn{5}{|l|}{ Female } \\
\hline Age, years & $57.8 \pm 5.6$ & $57.8 \pm 6.7$ & $58.7 \pm 4.8$ & 0.834 \\
\hline \multirow{2}{*}{$\begin{array}{l}\text { Education, } \\
\text { years }\end{array}$} & $n=16$ & $n=25$ & $n=19$ & \multirow{2}{*}{$<.001^{\star *}+\dagger+$} \\
\hline & $13 \pm 2.7$ & $13.4 \pm 3.8$ & $8.9 \pm 4.4$ & \\
\hline MMSE score & $28.3 \pm 1.3$ & $27.3 \pm 1.8$ & $20.5 \pm 6.7$ & $\begin{array}{l}<.001 * \star *+\dagger \dagger \\
0.01\end{array}$ \\
\hline MoCA score & $26.6 \pm 3.2$ & $26.1 \pm 2.5$ & $17.1 \pm 7.1$ & $\stackrel{<}{<.001 * \star \star+\dagger \dagger}$ \\
\hline \multirow{2}{*}{$\begin{array}{l}\mathrm{CSF} A \beta_{1-42} \\
\mathrm{pg} / \mathrm{ml}\end{array}$} & $n=5$ & $n=13$ & $\mathrm{n}=17$ & \multirow[t]{2}{*}{$<0.05^{\star}$} \\
\hline & $1173.2 \pm 274.2$ & $872.1 \pm 380.5$ & $701.5 \pm 396.6$ & \\
\hline \multirow{2}{*}{$\begin{array}{l}\text { CSF tau, } \\
\mathrm{pg} / \mathrm{ml}\end{array}$} & $n=5$ & $n=13$ & $\mathrm{n}=17$ & \multirow[t]{2}{*}{0.1368} \\
\hline & $302.0 \pm 41.0$ & $326.8 \pm 222.6$ & $543.4 \pm 416.1$ & \\
\hline
\end{tabular}

To confirm the specificity of miR-150-5p in detecting DAT, we evaluated the expression pattern of miR150-3p, a mature form of miR-150 but is not differentially expressed by miRNA-seq analysis, to serve as a negative control. No significant differences were observed among the three cohorts with regards to the expression pattern (Fig. 2c) and with AUC less than 0.60 (Fig. 2d).

\section{Higher miR-150-5p expression correlates with lower cognitive performance, lower CSF $A \beta_{1-42}$ and higher CSF tau}

To further confirm if miR-150-5p is a reliable biomarker for DAT, we studied the relationship between the elevated levels of miR-150-5p and the clinical cognitive performance outcome of the patients. MMSE and MoCA are commonly used clinical assessment for dementia[22, 23]. In both MMSE and MoCA, the lower 
the score, the more severe the cognitive impairment of patients are. Correlation analysis showed that expression levels of miR-150-5p correlated inversely with both MMSE $(r=$

$-0.3968 ; p=0.001$, Fig. 3a) and MoCA scores $(r=-0.4091 ; p=0.001$, Fig. 3b), that a higher miR-150-5p expression levels in PBMC was associated with lower MMSE and MoCA scores. Our results indicate that the higher miR-150-5p expression levels in PBMC is associated with poor cognitive outcome of the patient, which is consistent with the clinical diagnosis.

Next, we conducted correlation study between miRNA expression with AD pathology diagnosis. CSF $A \beta_{1-42}$ levels have been previously demonstrated to be lower in patients with pathologically confirmed DAT, compared to HS [33]. We found that there is a negative correlation between miR-150-5p expression levels and CSF A $\beta_{1-42}$ levels $(r=-0.3516, p=0.0355$, Fig. 3c). The increased miRNA expression associated with decreased $A \beta_{1-42}$ is consistent with the higher expression of miR-150-5p in DAT cohort (Fig. 2a). In addition, we found a positive correlation between miRNA expression and CSF tau levels, that the increased miR-150-5p is associated with increased CSF tau, $(r=0.3317, p=0.0553$, Fig. $3 d)$, indicating that higher miR-150-5p expression levels is associated with greater neurodegeneration.

\section{Higher miR-150-5p levels are associated with lower voxel- wise grey matter volume in $A D$ specific brain regions}

To study the correlation between miR-150-5p and AD brain atrophy patterns, we examined the influence of miR-150-5p expression levels on in vivo MRI-based brain structural measures involving whole-brain grey matter volume. We carried out whole-brain voxel-based morphometry analyses to assess voxel-wise grey matter differences between individuals with low and high miR-150-5p. We found that participants with higher than median levels of miR-150-5p showed lower voxel-wise grey matter volume primarily in parietal and temporal regions involving the medial temporal lobe, posterior cingulate cortex, and precuneus (FWE corrected $p<0.05$, Fig. 4a) when compared to participants with lower than median levels of miR-150-5p. On the other hand, participants with lower than median miR-150-5p levels did not show reduced voxel-wise grey matter volume compared to participants with higher than median miR-150-5p levels. To further investigate miR-150-5p influence on grey matter volume, we carried out a Pearson's correlation analyses between miR-150-5p expression levels and grey matter volume from specific regionsof-interest comprising the default mode network and executive control networks, two major networks targeted in AD. We found that higher miR-150-5p levels are associated with lower grey matter volume in the default mode network regions comprising the left hippocampus (Fig. 4b), right hippocampus (Fig. 4c), medial prefrontal cortex, posterior cingulate cortex (FWE corrected $p<0.0055)$ and precuneus (uncorrected $p=0.007$ ). Additionally, higher miR-150-5p levels are associated with lower grey matter volume in the executive control network regions comprising the left dorsolateral prefrontal cortex, right dorsolateral prefrontal cortex, left posterior cingulate cortex (Fig. 4d) and right posterior parietal cortex (Fig. 4e) at the FWE-corrected $p<0.0055$ threshold. Our result demonstrated that miR-150-5p is able to detect the cerebral atrophy in $A D$ specific regions. 


\section{MiR-150-5p targets are involved in Wnt signaling pathway that is linked to AD pathobiology}

To capture the molecular mechanisms that underlie the dysregulation of miR-150-5p in AD, we first, identified potential targets of miR-150-5p using three widely used miRNA target prediction algorithms (miRDB, Insect Genome and TargetScan). 120 common targets of miR-150-5p were revealed and were then subjected to Gene Ontology (GO) categories analysis to identify the functional role of miRNA targets. GO enrichment analysis showed that miRNAs regulate its targets negatively and is required for protein binding, as well as were enriched in nucleocytoplasm regions (Fig. 5a). In addition, we mapped 120 predicted miRNA targets in KEGG pathway using DAVID 6.8 to further identify miRNA targets in biological pathways (Fig. 5b). Next, we further narrowed down 11 candidate miR-150-5p target genes based on the overlapping genes between enriched GO categories and KEGG pathways with FDR $<0.06$ and found that these genes were related to $A D$ and/or Wnt signaling pathway (Fig. $5 \mathrm{c}$ ). Among these 11 genes, we focus on programmed cell death 4 (PDCD4) because it is a multifunctional tumor suppressor which regulates cell apoptosis, cell growth, protein translation and signal transduction, and impairs PI3K/AKT signaling pathway in cellular AD models[34, 35]. qPCR revealed that $P D C D 4$ was significantly downregulated in DAT compared to $\mathrm{MCl}$, which is opposite to that of miR-150-5p (Fig. $5 \mathrm{~d}$ ). In addition, we found that there are miR-150-5p putative binding sites (TTGGGAT) located at the $3^{\prime} U T R$ region of its mRNA (Fig. 5e). To further confirm the PDCD4 is the target of miR-150-5p, we conduct the cell-based analysis. Co-expression of miR-150-5p with 3'UTR of PDCD4 in HEK 293T cells significantly downregulates the PDCD4 at the transcription level by luciferase assay (Fig. $5 f$ and $g$ ). We further showed that expression of miR-150-5p in SH-SY5Y cells reduced PDCD4 at the mRNA level by qPCR assay (Fig. 5h), and at protein levels by western blot analysis (Fig. 5i). Therefore, PDCD4 is a potential downstream target of miR-150-5p, which may be related to miR-150-5p-associated pathogenesis in AD.

\section{Discussion}

In the present study, we systematically identified a potential PBMC-based miRNA biomarker for DAT. Our initial miRNA profiling allowed the identification of miRNAs with high differential expression in DAT and $\mathrm{MCl}$ individuals compared to HS individuals which was consistently upregulated in the discovery cohort and independent cohorts. Expression levels of miR-150-5p was upregulated in PBMC of patients with DAT and was closely correlated to cognitive performance, CSF $A \beta_{1-42}$ and tau levels, as well as MRI AD pattern of atrophy. Our results demonstrated that higher levels of miR-150-5p was associated with derogatory effects on brain structure, especially in regions of the brain belonging to the default mode and executive control networks, key brain networks implicated in AD. Furthermore, miR-150-5p was subjected to miRNA targets prediction, functional enrichment analysis, and verification of miRNA targets expression. Our bioinformatics analysis revealed that selected targets of miR-150-5p were markedly associated with Wnt signaling pathway which has been implicated in AD [36]. We further showed that target genes PDCD4 expression levels were downregulated in DAT. These results provide evidence that 
upregulation of miR-150-5p suppresses its downstream target genes. Therefore, our findings suggest that miR-150-5p is a potential as a biomarker for the diagnosis of DAT.

A previous study showed that PBMC is involved in the pathogenesis of $A D$ due to its central role in inflammation during the early phase of $A D$ development [37]. Lymphocytes and monocytes, which represent the majority of immune cells in PBMC, can infiltrate blood brain barrier (BBB) and lead to inflammatory cascade in the brain of $A D$ [38]. Furthermore, it is also reported that dysfunction of $B B B$ in $A D$ enables exchange of miRNAs between the brain and peripheral blood [39]. A recent review reported that a higher correlation of transcriptomic changes (pattern of gene expression) and epigenomic changes (pattern of DNA methylation) in the CNS and blood [40]. Taken together, it is hypothesized that that changes in the expression of miRNAs in the PBMC could reflect the neuropathogenic changes in the CNS and PBMC may serve as a potential source to find miRNAs signatures that could help to discriminate cognitively healthy subjects from diseased patients. To explore the role of PBMC derived miRNAs for DAT, we unbiasedly profiled the miRNAs from PBMC of 54 study participants. (Fig. 1a). Additionally, we validated the aberrantly expressed miRNA in an independent cohort of 64 study participants. As verified by qPCR, miR-150-5p was upregulated in DAT versus HS individuals and DAT versus $\mathrm{MCl}$ individuals with an AUC of 0.86 and 0.86 , respectively (Fig. $2 a$ and b).

In addition to differential expression of miRNA in $\mathrm{HS}, \mathrm{MCl}$, and DAT individuals, another important consideration is the reliability of the miRNA with regards to cognitive, neuroimaging, and CSF biomarkers for AD. In this regard, we observed miR-150-5p expression levels to be inversely correlated to cognitive tests including MMSE and MoCA, demonstrating that higher miR-150-5p levels was associated with lower cognitive scores (higher disease severity) (Fig. 3a and b). Our findings also demonstrate that miR-150-5p expression levels were negatively correlated with $C S F A \beta_{1-42}$ levels and positively correlated with $C S F$ tau levels (Fig. $3 \mathrm{c}$ and d). In addition, subjects with DAT also had lower CSF $A \beta_{1-42}$ and higher CSF tau compared to $\mathrm{HS}$ and $\mathrm{MCl}$ subjects. As expected, subjects with $\mathrm{MCl}$ had intermediate levels of both markers (Table 2). These findings further support the concept that CSF is the gold standard of AD diagnosis where cognitive impaired $A D$ patients with a low concentration of CSF $A \beta_{1-42}$ are usually accompanied by a higher concentration of CSF tau levels [41]. Furthermore, the concentration of CSF $A \beta_{1-42}$ in DAT was significantly higher compared to $\mathrm{MCl}$ subjects but was comparable to $\mathrm{MCl}$ subjects. Similar finding was found in the expression of miR-150-5p among three groups. Thus, not only miR-150$5 p$ expression differentiates DAT from HS, but it also correlates with cognitive performance and CSF $A \beta_{1-42}$ as well as CSF tau levels and thus has wide clinical implications.

In order to validate miRNAs as biomarkers of $A D$, there is a need to understand their relationship with in vivo markers of brain structure and $A D$ neuropathology such as amyloid- $\beta$ and tau. However, there is currently limited evidence illustrating the association between miRNA levels and brain structure in AD. Thus, how miRNA levels influence and relate to in vivo measures of brain structure and function in $A D$ remain a key gap. In this regard, our findings illustrate a derogatory influence of miR-150-5p levels on both voxel-wise and regional grey matter volume in DAT (Fig. 4). Prior studies suggest that effects of 
miRNA levels on neuropathological markers of AD including amyloid- $\beta$ and phosphorylated tau could be related to such changes in brain structure. For instance, one study illustrated high accuracy in the diagnosis of prodromal AD using PBMC miRNA markers in accordance with amyloid- $\beta$ deposition as observed on PET imaging [42]. Evidence also indicates that miRNAs regulate genes that are responsible for amyloid- $\beta$ and phosphorylated tau up-regulation with increasing evidence suggesting that miRNAs influence amyloid- $\beta$ production [15]. Additionally, prior studies show alterations of miRNA levels primarily in the grey matter such as those involving the temporal lobes of the brain which are also key regions of amyloid- $\beta$ and phosphorylated tau deposition in AD [43-45]. Studies in post-mortem human tissue illustrate miRNA expression correlates with the presence of early AD-related pathology in grey matter, specifically with the density of diffuse amyloid- $\beta$ plaques [45]. Such findings support the notion that patterns of miRNA expression in the cortical grey matter may contribute to, and explain underlying $A D$ pathogenesis $[45,46]$.

The presence of amyloid- $\beta$ plaques likely further initiate or potentiate downstream changes that culminate in neurofibrillary tau pathology, cell death, synapse elimination, and eventually cognitive impairment in individuals with altered miRNA levels [45]. Expression of various miRNAs can thus form important candidates in understanding disease progression and modulation in AD. Such events as well as miRNA influence on synaptic loss and synaptic damage are possible mechanisms underlying grey matter loss especially within the hippocampus as well as cognitive decline related to AD. While our group is one of the first to show associations between brain changes and miRNA levels, nonetheless, much remains to be explored regarding the relationship of miRNA levels and brain structure in regions known to be affected in AD.

A single miRNA is able to regulate multiple target mRNAs, and likewise a single target mRNA can be regulated by multiple miRNAs [47]. Using both target prediction and pathway enrichment approaches, we found out some miR-150-5p targets are enriched in the Wnt signaling, PI3K-AKT and thyroid hormone signaling pathways (Fig. 5b). Interestingly, these signaling pathways has been implicated in neurodevelopment and pathogenesis of $\operatorname{AD}[36,48,49]$. In addition, miR-150-5p targets were found enriched in the nucleus, nucleoplasm, nuclear membrane, cytosol, cytoplasmic mRNA processing body, and cytoplasm (Fig. 5c), which further demonstrated that the likelihood of miRNA targets shuttles between cytoplasm and nucleus.

It is noteworthy that miRNA negatively regulates the expression of its targets $[5,6]$. Consistently, miR-150$5 p$ targets, $P D C D 4$ were found to be downregulated in DAT compared to $\mathrm{MCl}$ and $\mathrm{HS}$ (Fig. 5d), which reflected the most enriched category for $\mathrm{GO}$ biological process that miR-150-5p is a negative regulator (Fig. 5a). PDCD4 (programmed cell death 4), has been demonstrated to be an important tumour suppressor in various cancers by inducing cell apoptosis. Until recently, PDCD4 was found to be regulated by miR-21 via PI3K/AKT signaling pathway in in vitro model of AD [34]. In the treatment of SH-SY5Y cells with $A \beta_{1-42}, P D C D 4$ expression were repressed by miR-21. In this study, our results validated that PDCD4 was remarkably reduced in DAT individuals. Through luciferase assay, we identified that miR-150-5p 
suppressed expression of $P D C D 4$ at the transcription level. So far, little is known about the biological role of $P D C D 4$ in miR-150-5p-based regulatory mechanisms of AD. Further investigation is warranted.

\section{Conclusions}

Our findings identified miR-150-5p as a potential candidate biomarker for DAT. miR-150-5p was well correlated with CSF $A \beta_{1-42}$ and tau, $A D$ pattern of cerebral atrophy and cognitive performance. To the best of our knowledge, this study is the first to demonstrate the role of miR-150-5p as a potential biomarker of DAT coupled with clinical measures (Fig. 6). While miR-150-5p has the potential of being developed as a clinical biomarker for DAT, future longitudinal studies with larger cohorts are needed to further validate the utility of miR-150-5p in DAT.

\section{Abbreviations}

$A \beta=$ amyloid $-\beta ;$ PBMC = peripheral blood mononuclear cells; DAT = dementia of Alzheimer's disease type; MMSE $=$ Mini-Mental State Examination; $\mathrm{MoCA}=$ Montreal Cognitive Assessment; $\mathrm{MCl}=$ mild cognitive impairment

\section{Declarations}

\section{Acknowledgments}

We acknowledge Christina Lim for the blood samples processing.

\section{Author's Contributions}

S.Y.C., A.V., K.P.N., A.S.B., H.T., B.Z.W., Y.J.T., and F. Z. performed the study and acquired, analysed, interpretedthe data, draft and edited the manuscript. A.S.N., S.C.L., K.O., and E.K.T analysed and interpreted the data. N.K. and L.Z conceived and designed the study, acquired, analysed, interpreted the data and drafted the manuscript with input from the co-authors.

\section{Funding}

This research was supported by National Neuroscience Institute Centre Grant, NMRC Individual Research Grant and Singapore Translational Research Investigator (STaR) award grant.

\section{Availability of data and materials}

All data associated with this study are available upon request.

\section{Ethics approval and consent to participate}

The study was approved by the SingHealth Centralized Review Board and adhered to the tenets of the Declaration of Helsinki and local clinical research regulations. A signed informed consent was also 
obtained from each patient.

Consent for publication

Not applicable.

Competing Interests

Authors declare that they have no competing interests.

\section{References}

1. Jack CR, Jr., Bennett DA, Blennow K, Carrillo MC, Dunn B, Haeberlein SB, Holtzman DM, Jagust W, Jessen F, Karlawish J, et al: NIA-AA Research Framework: Toward a biological definition of Alzheimer's disease. Alzheimers Dement 2018, 14:535-562.

2. McKhann GM, Knopman DS, Chertkow H, Hyman BT, Jack CR, Jr., Kawas CH, Klunk WE, Koroshetz WJ, Manly JJ, Mayeux R, et al: The diagnosis of dementia due to Alzheimer's disease: recommendations from the National Institute on Aging-Alzheimer's Association workgroups on diagnostic guidelines for Alzheimer's disease. Alzheimers Dement 2011, 7:263-269.

3. Khan TK, Alkon DL: Alzheimer's Disease Cerebrospinal Fluid and Neuroimaging Biomarkers: Diagnostic Accuracy and Relationship to Drug Efficacy. J Alzheimers Dis 2015, 46:817-836.

4. Shaw LM, Vanderstichele H, Knapik-Czajka M, Clark CM, Aisen PS, Petersen RC, Blennow K, Soares H, Simon A, Lewczuk P, et al: Cerebrospinal fluid biomarker signature in Alzheimer's disease neuroimaging initiative subjects. Ann Neurol 2009, 65:403-413.

5. Lausted C, Lee I, Zhou Y, Qin S, Sung J, Price ND, Hood L, Wang K: Systems approach to neurodegenerative disease biomarker discovery. Annu Rev Pharmacol Toxicol 2014, 54:457-481.

6. Persengiev SP, Kondova, II, Bontrop RE: The Impact of MicroRNAs on Brain Aging and Neurodegeneration. Curr Gerontol Geriatr Res 2012, 2012:359369.

7. Vreugdenhil E, Berezikov E: Fine-tuning the brain: MicroRNAs. Frontiers in neuroendocrinology 2010, 31:128-133.

8. Cao DD, Li L, Chan WY: MicroRNAs: Key Regulators in the Central Nervous System and Their Implication in Neurological Diseases. Int J Mol Sci 2016, 17.

9. Banning LCP, Ramakers I, Deckers K, Verhey FRJ, Aalten P: Affective symptoms and AT(N) biomarkers in mild cognitive impairment and Alzheimer's disease: A systematic literature review. Neurosci Biobehav Rev 2019, 107:346-359.

10. Martinez B, Peplow PV: MicroRNAs as diagnostic and therapeutic tools for Alzheimer's disease: advances and limitations. Neural Regen Res 2019, 14:242-255.

11. Piscopo P, Lacorte E, Feligioni M, Mayer F, Crestini A, Piccolo L, Bacigalupo I, Filareti M, Ficulle E, Confaloni A, et al: MicroRNAs and mild cognitive impairment: A systematic review. Ageing Res Rev 2019, 50:131-141. 
12. Zhang W, Thevapriya S, Kim PJ, Yu WP, Je HS, Tan EK, Zeng L: Amyloid precursor protein regulates neurogenesis by antagonizing miR-574-5p in the developing cerebral cortex. Nat Commun 2014, 5:3330.

13. Zhang W, Kim PJ, Chen Z, Lokman H, Qiu L, Zhang K, Rozen SG, Tan EK, Je HS, Zeng L: MiRNA-128 regulates the proliferation and neurogenesis of neural precursors by targeting PCM1 in the developing cortex. Elife 2016, 5.

14. Kumar S, Reddy PH: Are circulating microRNAs peripheral biomarkers for Alzheimer's disease? Biochim Biophys Acta 2016, 1862:1617-1627.

15. Reddy PH, Williams J, Smith F, Bhatti JS, Kumar S, Vijayan M, Kandimalla R, Kuruva CS, Wang R, Manczak M, et al: MicroRNAs, Aging, Cellular Senescence, and Alzheimer's Disease. Prog Mol Biol Trans/ Sci 2017, 146:127-171.

16. Albert MS, DeKosky ST, Dickson D, Dubois B, Feldman HH, Fox NC, Gamst A, Holtzman DM, Jagust WJ, Petersen RC, et al: The diagnosis of mild cognitive impairment due to Alzheimer's disease: recommendations from the National Institute on Aging-Alzheimer's Association workgroups on diagnostic guidelines for Alzheimer's disease. Alzheimers Dement 2011, 7:270-279.

17. Sperling RA, Aisen PS, Beckett LA, Bennett DA, Craft S, Fagan AM, Iwatsubo T, Jack CR, Jr., Kaye J, Montine TJ, et al: Toward defining the preclinical stages of Alzheimer's disease: recommendations from the National Institute on Aging-Alzheimer's Association workgroups on diagnostic guidelines for Alzheimer's disease. Alzheimers Dement 2011, 7:280-292.

18. Ha M, Kim VN: Regulation of microRNA biogenesis. Nat Rev Mol Cell Bio/ 2014, 15:509-524.

19. Swarbrick S, Wragg N, Ghosh S, Stolzing A: Systematic Review of miRNA as Biomarkers in Alzheimer's Disease. Mol Neurobio/ 2019, 56:6156-6167.

20. Boeckel JN, Thome CE, Leistner D, Zeiher AM, Fichtlscherer S, Dimmeler S: Heparin selectively affects the quantification of microRNAs in human blood samples. Clin Chem 2013, 59:1125-1127.

21. Williams MM, Storandt M, Roe CM, Morris JC: Progression of Alzheimer's disease as measured by Clinical Dementia Rating Sum of Boxes scores. Alzheimers Dement 2013, 9:S39-44.

22. Folstein MF, Folstein SE, McHugh PR: "Mini-mental state". A practical method for grading the cognitive state of patients for the clinician. J Psychiatr Res 1975, 12:189-198.

23. Ng A, Chew I, Narasimhalu K, Kandiah N: Effectiveness of Montreal Cognitive Assessment for the diagnosis of mild cognitive impairment and mild Alzheimer's disease in Singapore. Singapore Med $J$ 2013, 54:616-619.

24. Blennow K, Chen C, Cicognola C, Wildsmith KR, Manser PT, Bohorquez SMS, Zhang Z, Xie B, Peng J, Hansson O, et al: Cerebrospinal fluid tau fragment correlates with tau PET: a candidate biomarker for tangle pathology. Brain 2020, 143:650-660.

25. Maes OC, Xu S, Yu B, Chertkow HM, Wang E, Schipper HM: Transcriptional profiling of Alzheimer blood mononuclear cells by microarray. Neurobiol Aging 2007, 28:1795-1809.

26. Ashburner J, Friston KJ: Unified segmentation. Neuroimage 2005, 26:839-851. 
27. Ashburner J: A fast diffeomorphic image registration algorithm. Neuroimage 2007, 38:95-113.

28. Shirer WR, Ryali S, Rykhlevskaia E, Menon V, Greicius MD: Decoding subject-driven cognitive states with whole-brain connectivity patterns. Cereb Cortex 2012, 22:158-165.

29. Wang BZ, Zailan FZ, Wong BYX, Ng KP, Kandiah N: Identification of novel candidate autoantibodies in Alzheimer's disease. Eur J Neurol 2020.

30. Duan D, Li C, Shen L, Cui C, Shu T, Zheng J: Regional Gray Matter Atrophy Coexistent with Occipital Periventricular White Matter Hyper Intensities. Front Aging Neurosci 2016, 8:214.

31. Grau-Olivares M, Arboix A, Junque C, Arenaza-Urquijo EM, Rovira M, Bartres-Faz D: Progressive gray matter atrophy in lacunar patients with vascular mild cognitive impairment. Cerebrovasc Dis 2010, 30:157-166.

32. Lau P, Bossers K, Janky R, Salta E, Frigerio CS, Barbash S, Rothman R, Sierksma AS, Thathiah A, Greenberg $D$, et al: Alteration of the microRNA network during the progression of Alzheimer's disease. EMBO Mol Med 2013, 5:1613-1634.

33. Smith AD, Refsum H, Bottiglieri T, Fenech M, Hooshmand B, McCaddon A, Miller JW, Rosenberg IH, Obeid R: Homocysteine and Dementia: An International Consensus Statement. J Alzheimers Dis 2018, 62:561-570.

34. Feng MG, Liu CF, Chen L, Feng WB, Liu M, Hai H, Lu JM: MiR-21 attenuates apoptosis-triggered by amyloid-beta via modulating PDCD4/ PI3K/AKT/GSK-3beta pathway in SH-SY5Y cells. Biomed Pharmacother 2018, 101:1003-1007.

35. Zhang D, Shi Z, Li M, Mi J: Hypoxia-induced miR-424 decreases tumor sensitivity to chemotherapy by inhibiting apoptosis. Cell Death Dis 2014, 5:e1301.

36. Palomer E, Buechler J, Salinas PC: Wnt Signaling Deregulation in the Aging and Alzheimer's Brain. Front Cell Neurosci 2019, 13:227.

37. Kinney JW, Bemiller SM, Murtishaw AS, Leisgang AM, Salazar AM, Lamb BT: Inflammation as a central mechanism in Alzheimer's disease. Alzheimers Dement (N Y) 2018, 4:575-590.

38. Wojsiat J, Laskowska-Kaszub K, Mietelska-Porowska A, Wojda U: Search for Alzheimer's disease biomarkers in blood cells: hypotheses-driven approach. Biomark Med 2017, 11:917-931.

39. Zipser BD, Johanson CE, Gonzalez L, Berzin TM, Tavares R, Hulette CM, Vitek MP, Hovanesian V, Stopa EG: Microvascular injury and blood-brain barrier leakage in Alzheimer's disease. Neurobiol Aging 2007, 28:977-986.

40. Tylee DS, Kawaguchi DM, Glatt SJ: On the outside, looking in: a review and evaluation of the comparability of blood and brain "-omes". Am J Med Genet B Neuropsychiatr Genet 2013, 162B:595603.

41. Galasko D, Chang L, Motter R, Clark CM, Kaye J, Knopman D, Thomas R, Kholodenko D, Schenk D, Lieberburg I, et al: High cerebrospinal fluid tau and low amyloid beta42 levels in the clinical diagnosis of Alzheimer disease and relation to apolipoprotein E genotype. Arch Neurol 1998, 55:937-945. 
42. Cheng L, Doecke JD, Sharples RA, Villemagne VL, Fowler CJ, Rembach A, Martins RN, Rowe CC, Macaulay SL, Masters CL, et al: Prognostic serum miRNA biomarkers associated with Alzheimer's disease shows concordance with neuropsychological and neuroimaging assessment. Mol Psychiatry 2015, 20:1188-1196.

43. Braak H, Braak E: Neuropathological stageing of Alzheimer-related changes. Acta Neuropathol 1991, 82:239-259.

44. Thal DR, Rub U, Orantes $M$, Braak H: Phases of A beta-deposition in the human brain and its relevance for the development of AD. Neurology 2002, 58:1791-1800.

45. Wang WX, Huang Q, Hu Y, Stromberg AJ, Nelson PT: Patterns of microRNA expression in normal and early Alzheimer's disease human temporal cortex: white matter versus gray matter. Acta Neuropathol 2011, 121:193-205.

46. Wang WX, Rajeev BW, Stromberg AJ, Ren N, Tang G, Huang Q, Rigoutsos I, Nelson PT: The expression of microRNA miR-107 decreases early in Alzheimer's disease and may accelerate disease progression through regulation of beta-site amyloid precursor protein-cleaving enzyme 1 . J Neurosci 2008, 28:1213-1223.

47. Lewis BP, Shih IH, Jones-Rhoades MW, Bartel DP, Burge CB: Prediction of mammalian microRNA targets. Cell 2003, 115:787-798.

48. de Jong FJ, den Heijer T, Visser TJ, de Rijke YB, Drexhage HA, Hofman A, Breteler MM: Thyroid hormones, dementia, and atrophy of the medial temporal lobe. J Clin Endocrinol Metab 2006, 91:2569-2573.

49. Gabbouj S, Ryhänen S, Marttinen M, Wittrahm R, Takalo M, Kemppainen S, Martiskainen H, Tanila H, Haapasalo A, Hiltunen M, Natunen T: Altered Insulin Signaling in Alzheimer's Disease Brain - Special Emphasis on PI3K-Akt Pathway. Frontiers in Neuroscience 2019, 13.

50. Subramaniam M, Chong SA, Vaingankar JA, Abdin E, Chua BY, Chua HC, Eng GK, Heng D, Hia SB, Huang W, et al: Prevalence of Dementia in People Aged 60 Years and Above: Results from the WiSE Study. J Alzheimers Dis 2015, 45:1127-1138.

\section{Figures}


a

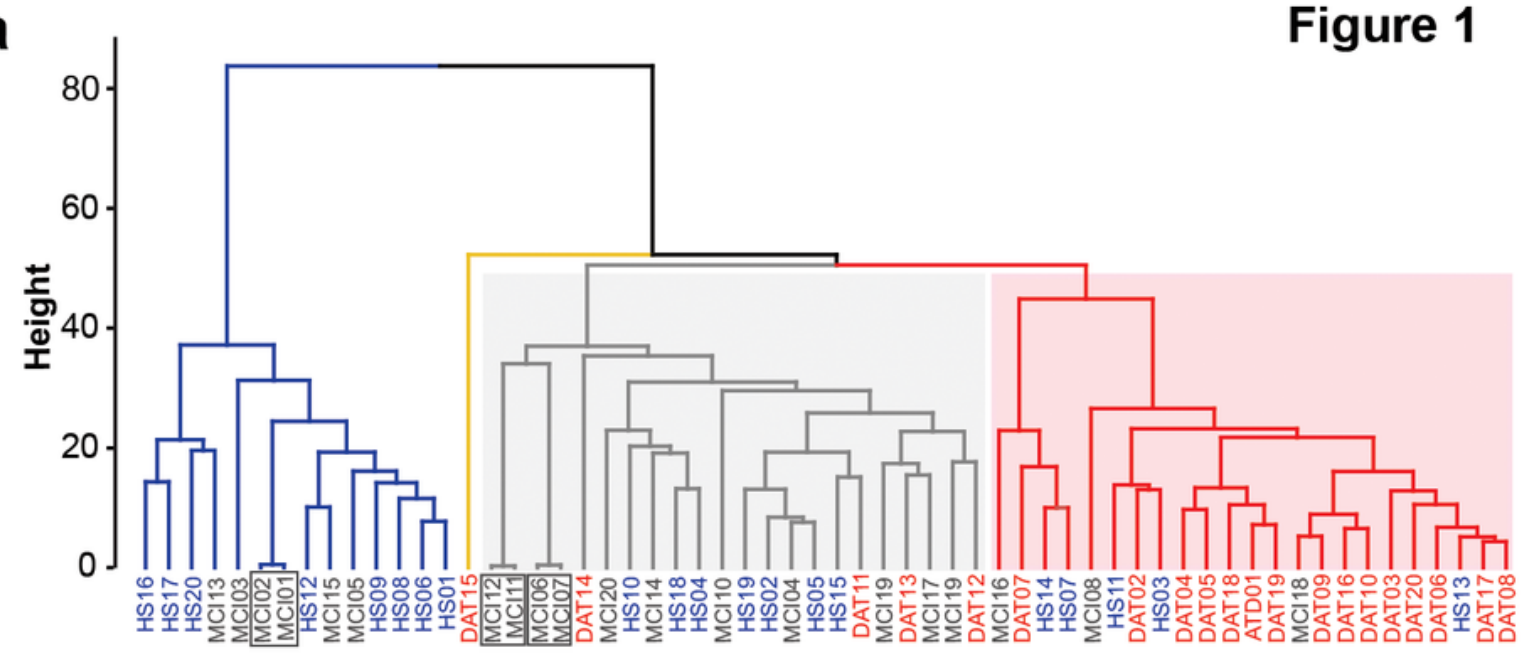

b

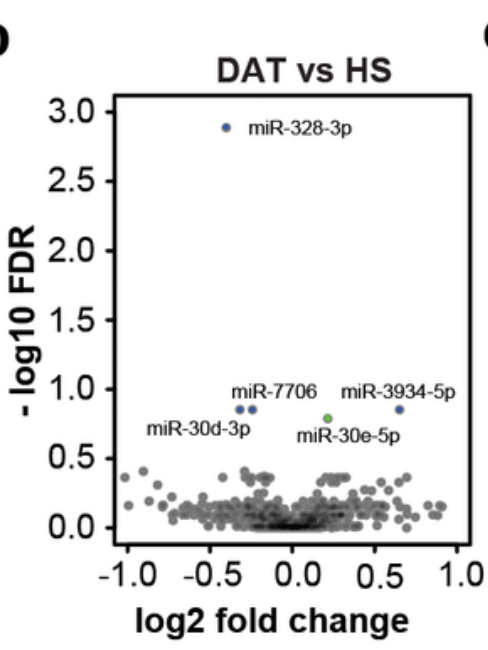

C

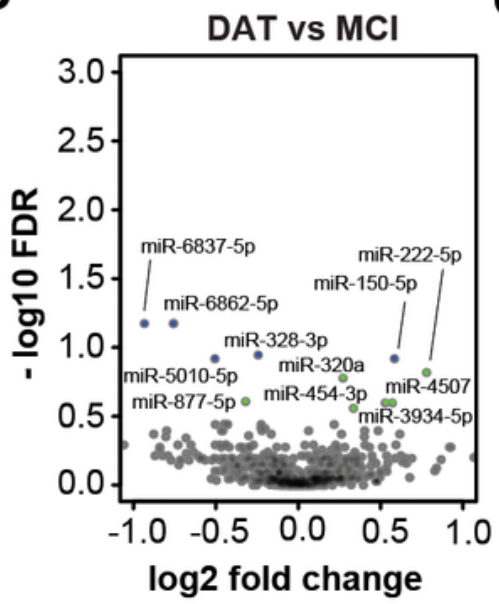

d

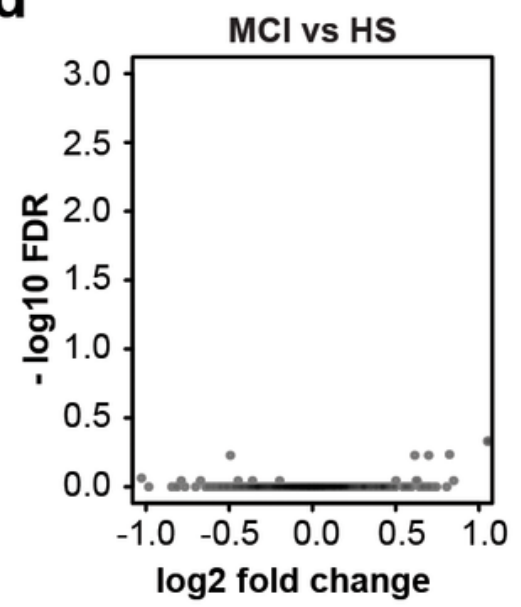

e

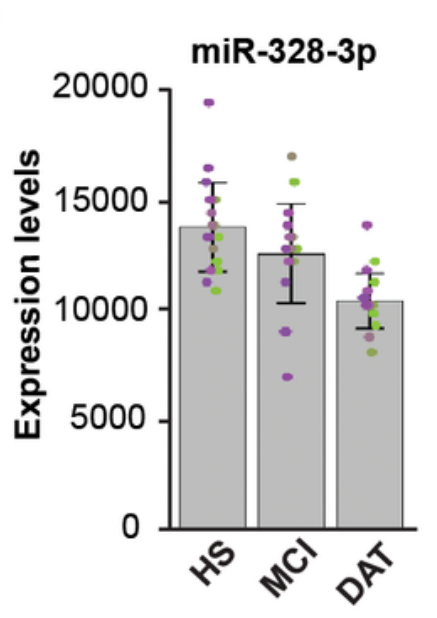

f

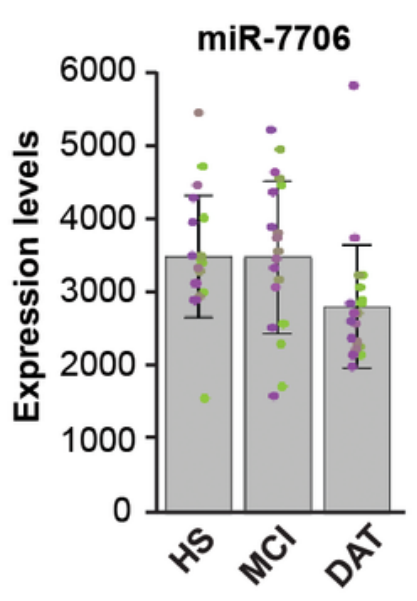

g

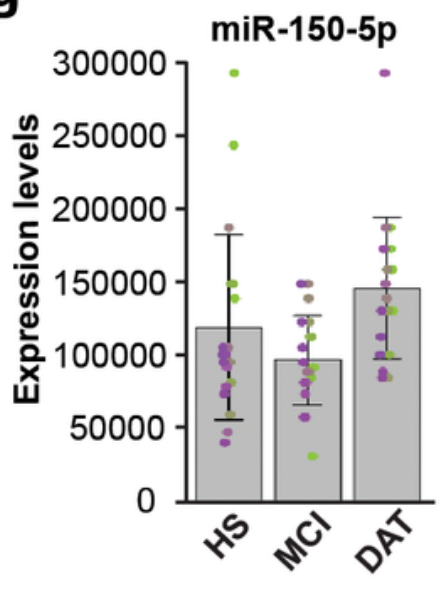

\section{Figure 1}

MiRNA-sequencing data analysis reveals Alzheimer's dementia is distinct from $\mathrm{MCl}$ and healthy subjects. (a) Hierarchical clustering analysis. $\mathrm{HS}, \mathrm{MCl}$ and DAT individuals from discovery cohort were labeled with blue, grey and red letters. The MC replicates sequenced on different lanes are marked by black rectangles, suggesting that the technical error was small. The dendrogram showed four clusters: blue, yellow, grey and red lines. DAT were enriched in the group shaded by the red rectangle. Volcano plots were drawn by 
plotting -log10 (FDR) against log2 (fold change) of each miRNA in comparison between (b) DAT and HS, (c) DAT and $\mathrm{MCl}$, and (d) $\mathrm{MCl}$ and $\mathrm{HS}$ subjects. Wilcoxon-Mann-Whitney (WMW) p-values with BenjaminiHochberg adjustment were used as FDR. Differentially expressed genes at $<15,30 \%$ cutoffs were colored by blue and green. Expression patterns of differentially expressed (e) miR-328-3p, (f) -7706 and (g) -150$5 p$. Dots represented individual expression values. Data shown as means \pm SD.

a

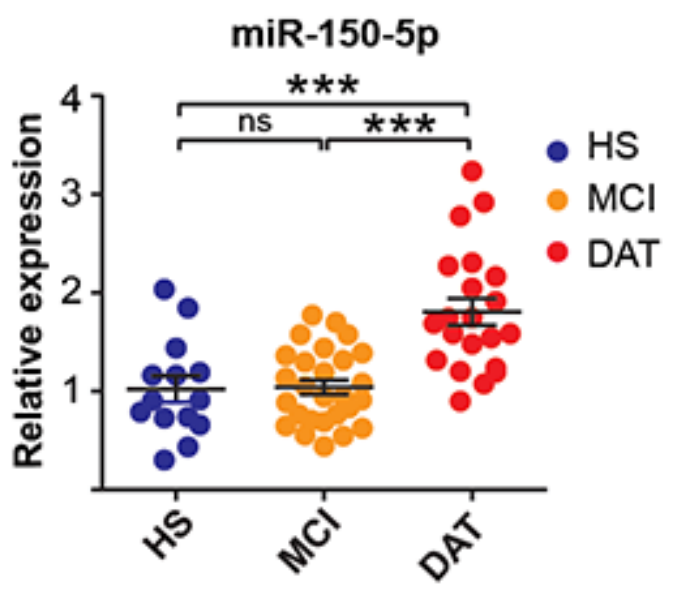

C

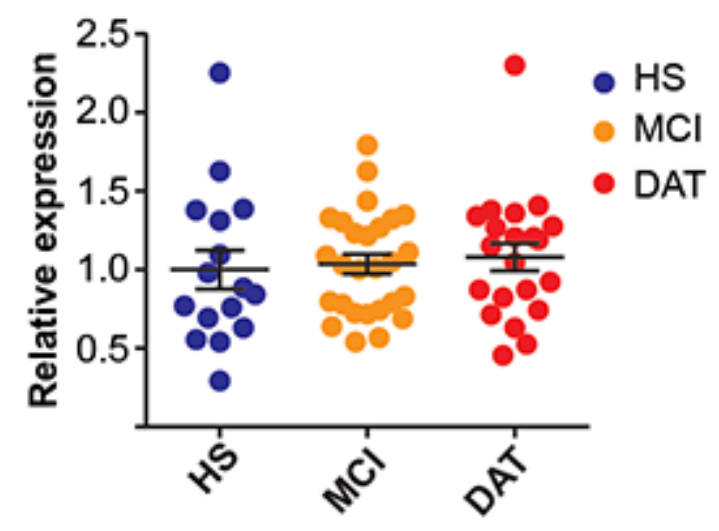

b

miR-150-5p

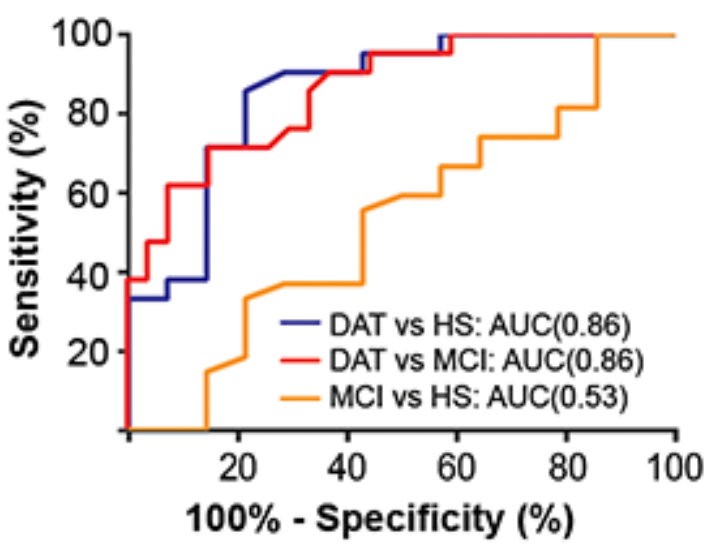

d

miR-150-3p

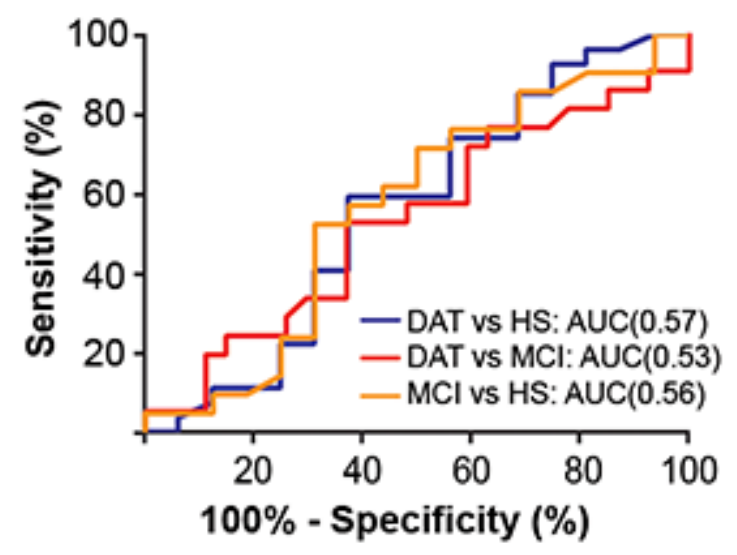

Figure 2

Figure 2

MiR-150-5p expression is upregulated in dementia of Alzheimer's disease type individuals. Expression levels (a) and receiving operating curve (ROC) analysis (b) of miR-150-5p in PBMC of an independent cohort. Expression levels (c) and ROC analysis (d) of miR-150-3p in PBMC of an independent cohort. Data are presented as the mean \pm SEM. One-way ANOVA with Tukey post hoc test was used to compare the differences between each disease group for all data sets. The statistical significance levels were set at $\star * \star p<0.001$, ns $=$ no significant. 
a

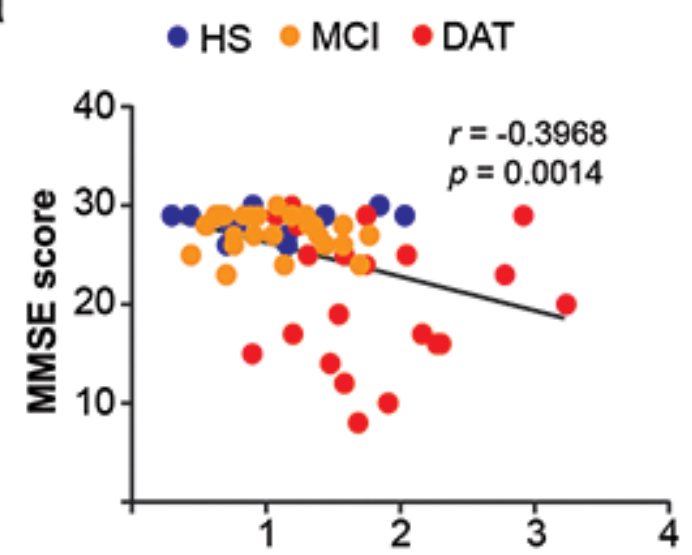

miR-150-5p relative expression

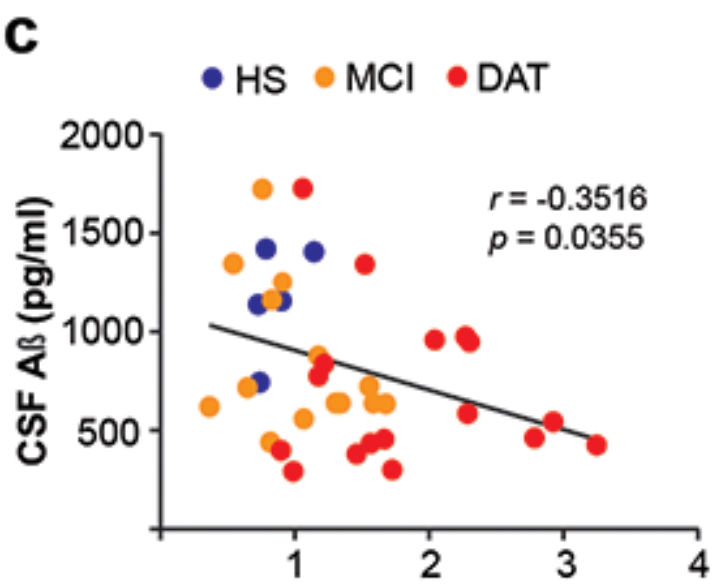

miR-150-5p relative expression b

$\bullet \mathrm{HS} \bullet \mathrm{MCl} \bullet \mathrm{DAT}$

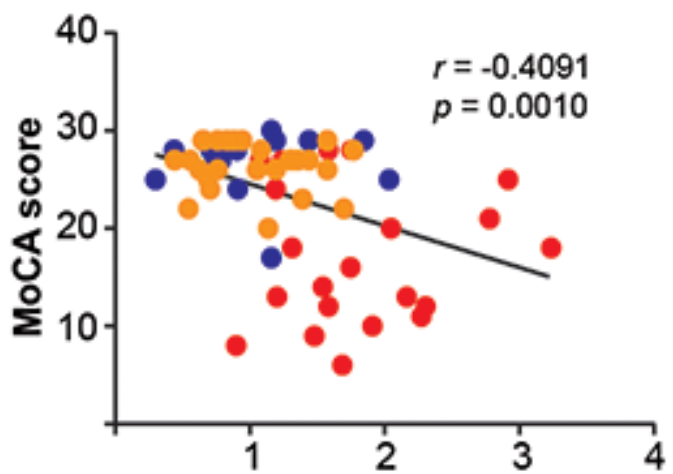

$\mathrm{miR}-150-5 \mathrm{p}$ relative expression

d

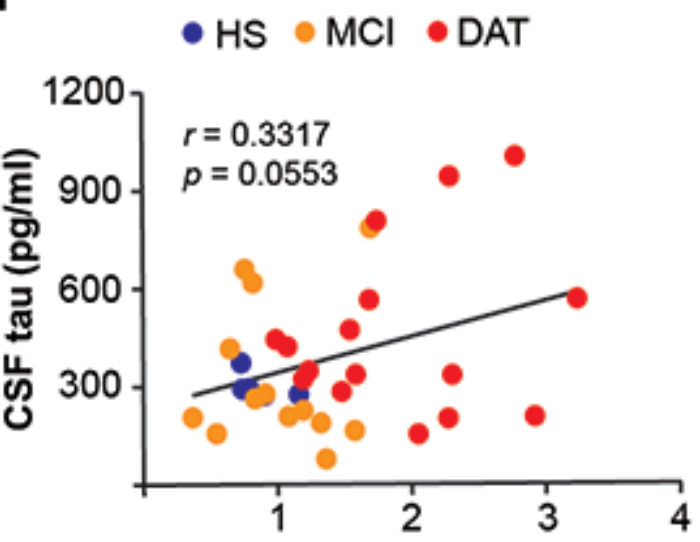

miR-150-5p relative expression

Figure 3

Figure 3

Association between expression levels of miR-150-5p and cognitive assessment, CSF A $1-42$ and tau levels. Correlations of miR-150-5p expression levels with (a) MMSE score and (b) MoCA score, (c) CSF A 1-42 levels, and (d) CSF tau levels are shown. Lower scores indicate cognitive decline. Lower CSF AB142 and higher CSF tau levels indicate higher risk of associated with dementia of the Alzheimer's type. Data were analyzed by a Spearman correlation test. The statistical significances were set at ${ }^{*} \mathrm{p}<0.01$ and $* \star \star p<0.001$. 


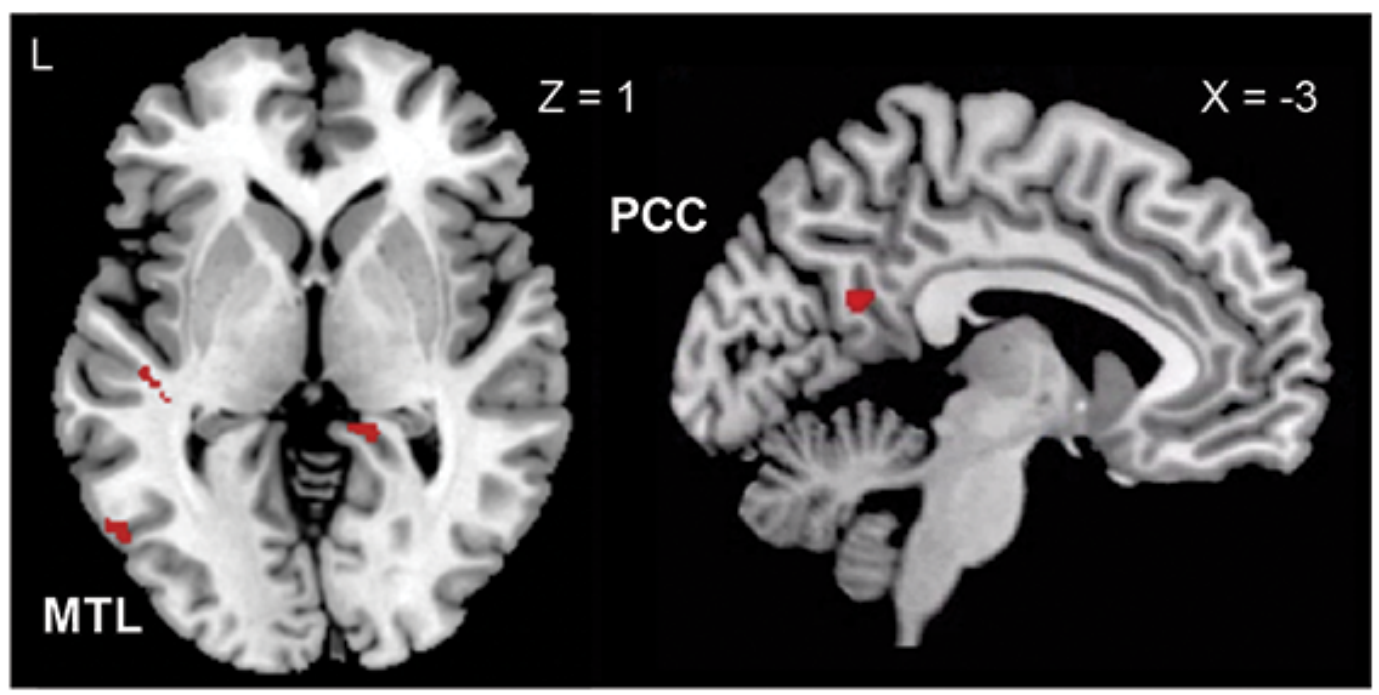

b

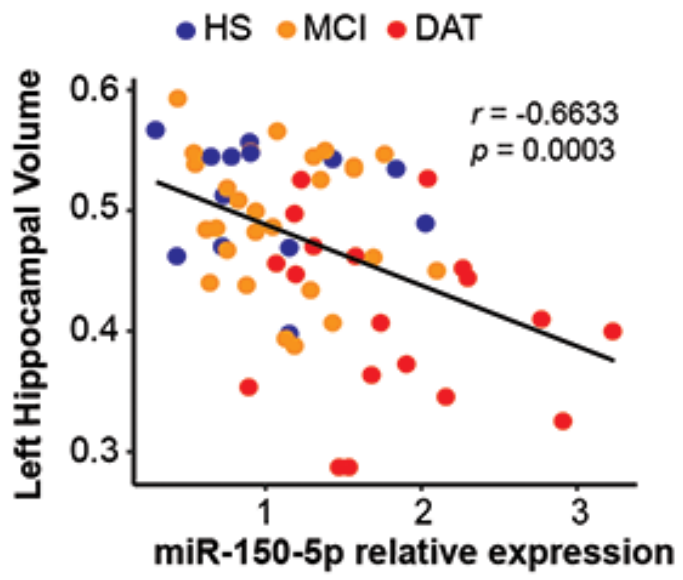

d

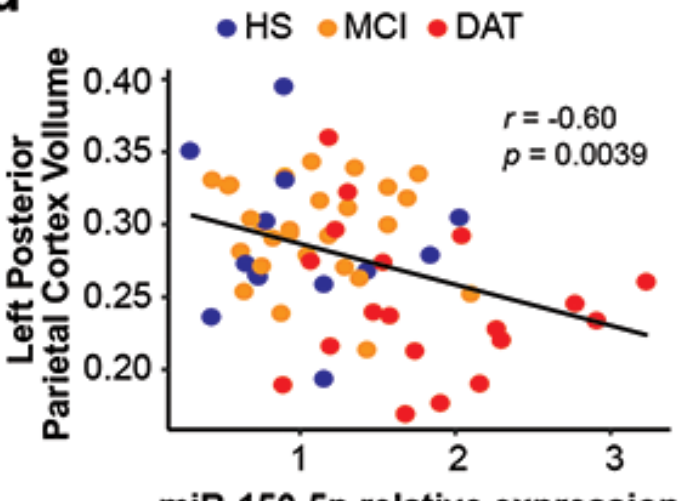

miR-150-5p relative expression
C

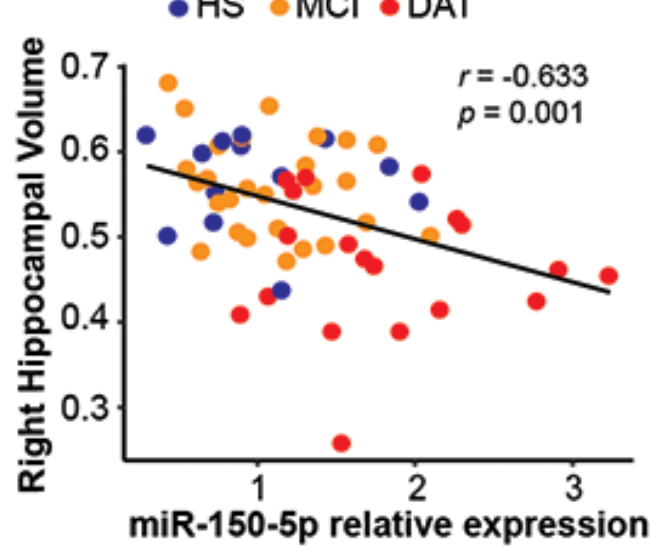

e

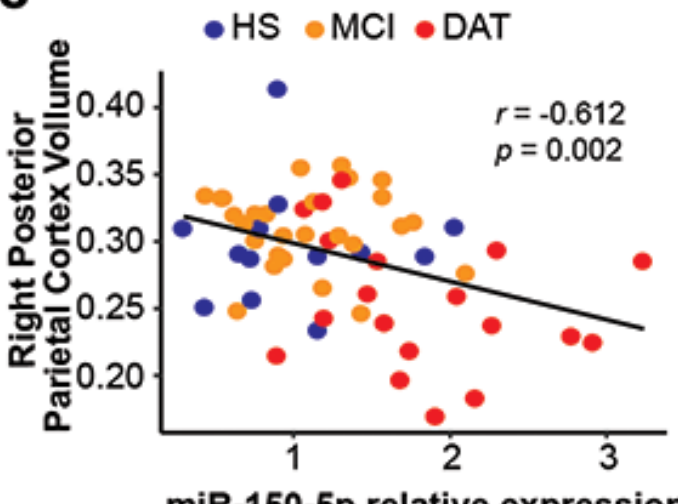

miR-150-5p relative expression

Figure 4

Figure 4

High miR-150-5p levels are associated with lower voxel-wise grey matter volume in AD specific regions. (a) Higher than median miRNA levels are associated with lower voxel-wise grey matter volume in temporal and parietal regions. Individuals with higher than median level of miR-150-5p showed reduced grey matter volume in parietal and temporal regions. (b-c) Higher miR-150-5p levels are associated with lower grey matter volume in the default mode network. Increasing levels of miR-150-5p were related to 
lower grey matter volume in the left and right hippocampal regions of interest comprising the default mode network. (d-e) Higher miR-150-5p levels are associated with lower grey matter volume in the executive control network. Increasing levels of miR-150-5p were related to lower grey matter volume in the left and right posterior parietal cortex regions of interest comprising the executive control network.

a

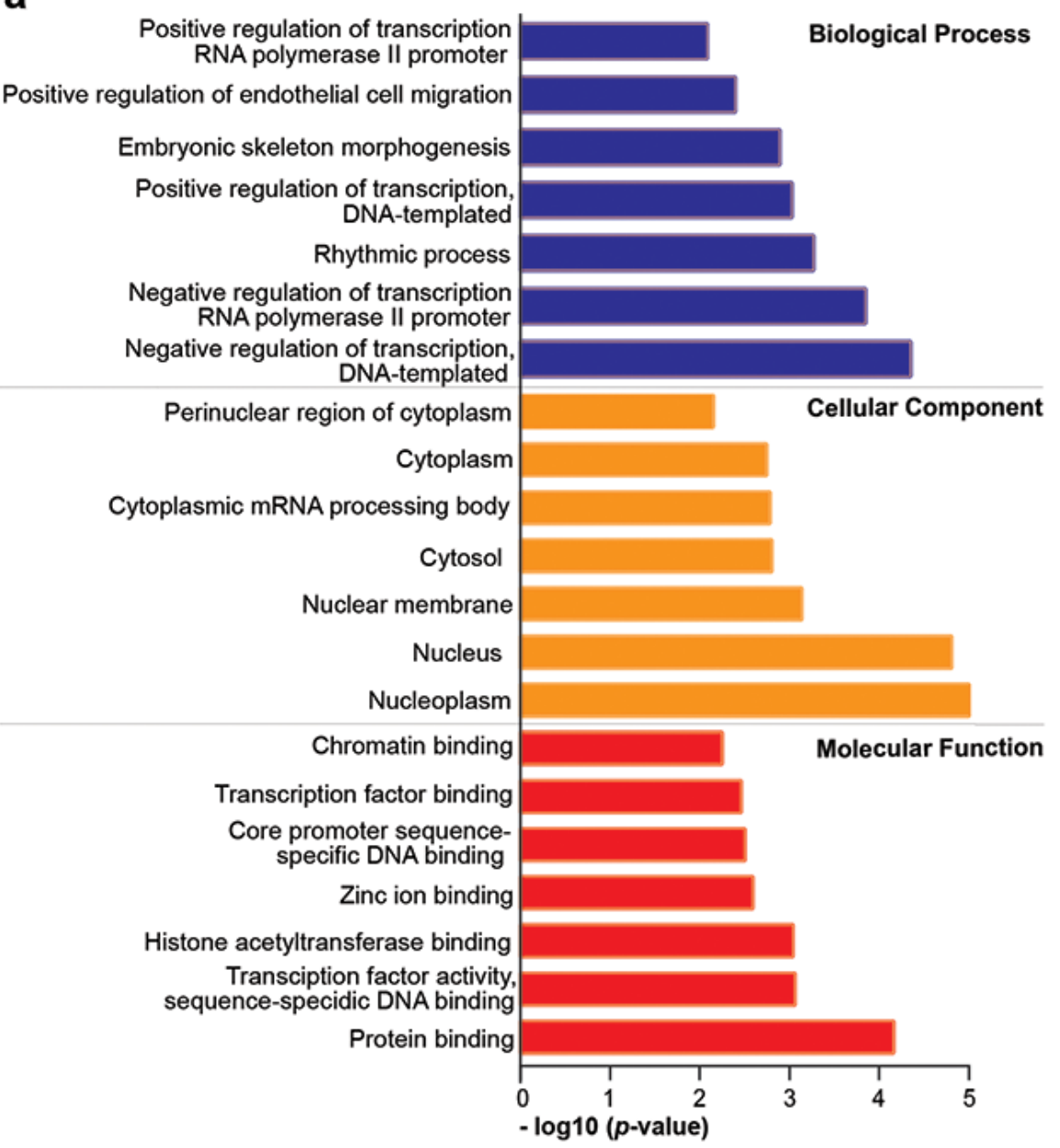

d

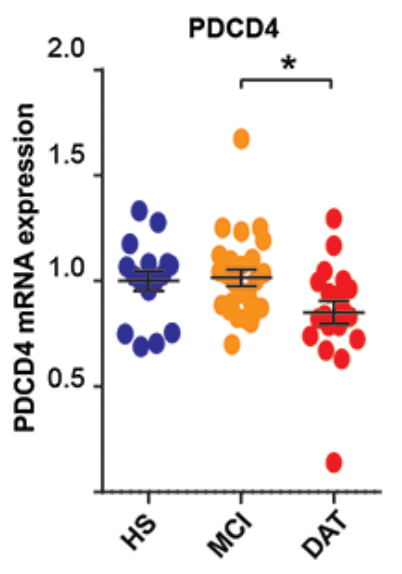

e

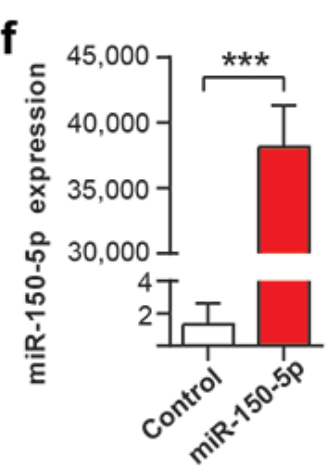

g

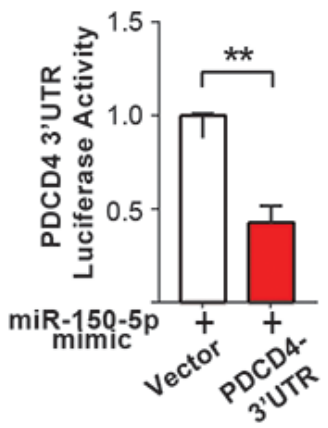

b

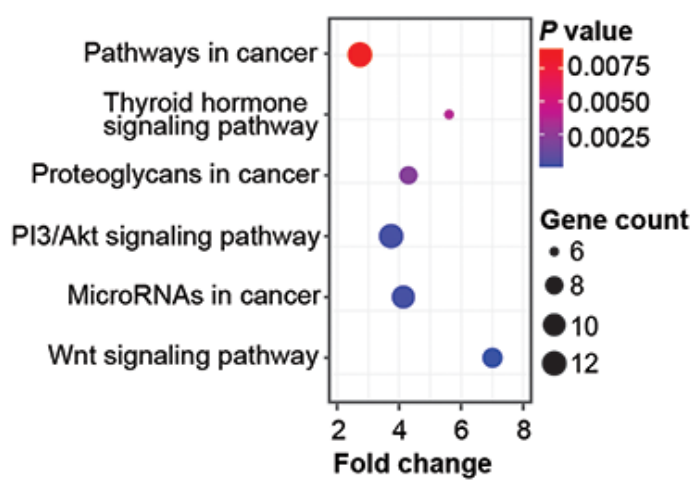

C

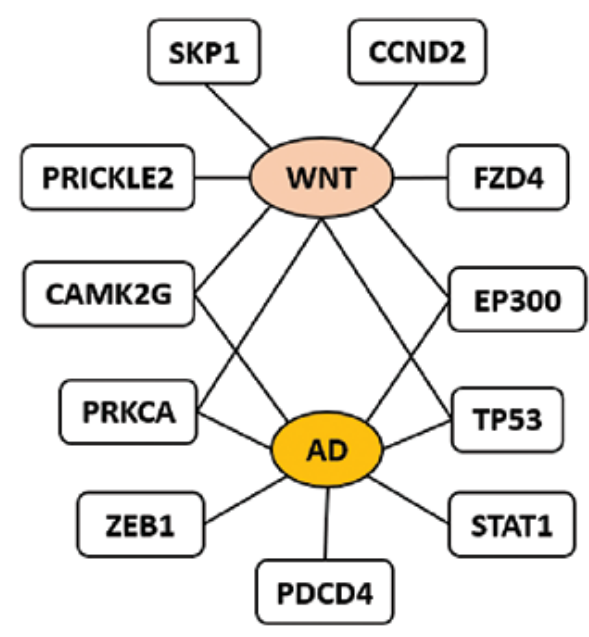

i

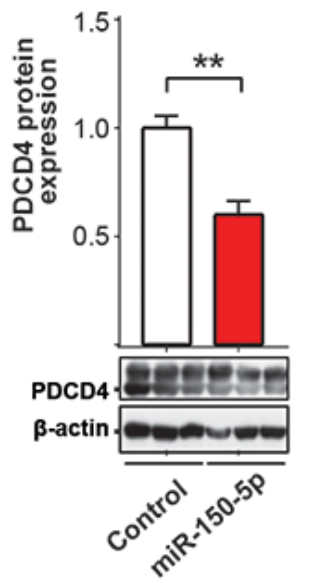

Figure 5

Figure 5 
PDCD4 is the downstream target of miR-150-5p. (a) Enriched GO terms for biological process, molecular function and cellular components [50]. (b) Enriched KEGG pathway analysis. Significantly enriched terms were presented with cut-off $p<0.01$. (c) Top 11 potential miR-150-5p targets which were implicated to AD pathology and/or Wnt signaling pathway. (d) Expression levels of PDCD4 in PBMC of an independent cohort. (e) One of the four putative miR-150-5p binding sites (AACCCUC-red), position 2081-2096 at the 3'UTR of PDCD4 shown. (f) Overexpression of miR-150-5p by miR-150-5p mimic. (g) Luciferase assay of PDCD4 upon miR-150-5p overexpression. (h-i) Overexpressed miR-150-5p downregulates PDCD4 $(h)$ mRNA by qPCR and (i) protein level by western blot. The statistical significances were set at ${ }^{*} p<0.05$, $* * p$ $<0.01$, and ${ }^{* *} \mathrm{p} p 0.001$ by student's t test.

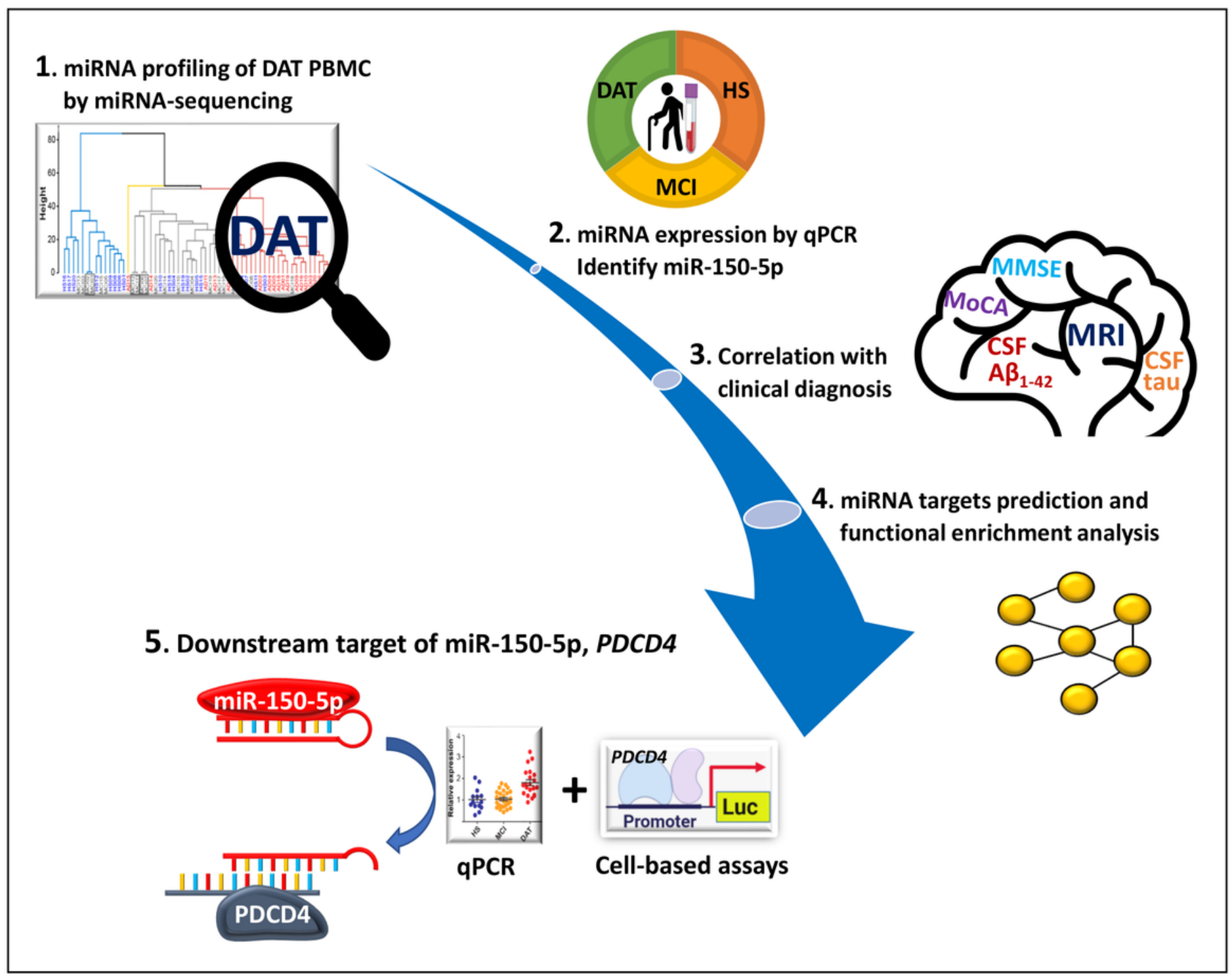

Figure 6

Schematic workflow for identification of miR-150-5p as a biomarker in PBMC of DAT. The schematic diagram describes the miRNA expression profile using miRNA-seq, followed by validation of predictive 
miR-150-5p in PBMC of an independent cohort. Subsequently, miR-150-5p was identified as a potential DAT biomarker by using clinical and experimental approaches. 